\title{
On scattering from the one-dimensional multiple Dirac delta potentials
}

\author{
Fatih Erman $^{1,4}{ }^{(\mathbb{D}}$, Manuel Gadella ${ }^{2}$ and Haydar Uncu ${ }^{3}$ \\ ${ }^{1}$ Department of Mathematics, İzmir Institute of Technology, Urla, 35430, İzmir, \\ Turkey \\ ${ }^{2}$ Departamento de Física Teórica, Atómica y Óptica and IMUVA. Universidad de \\ Valladolid, Campus Miguel Delibes, Paseo Belén 7, E-47011, Valladolid, Spain \\ ${ }^{3}$ Department of Physics, Adnan Menderes University, 09100, Aydın, Turkey \\ E-mail: fatih.erman@gmail.com, manuelgadella1@gmail.com and huncu@adu.edu.tr
}

Received 19 October 2017, revised 25 December 2017

Accepted for publication 18 January 2018

Published 16 March 2018

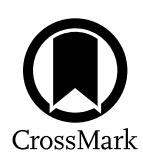

\begin{abstract}
In this paper, we propose a pedagogical presentation of the LippmannSchwinger equation as a powerful tool, so as to obtain important scattering information. In particular, we consider a one-dimensional system with a Schrödinger-type free Hamiltonian decorated with a sequence of $N$ attractive Dirac delta interactions. We first write the Lippmann-Schwinger equation for the system and then solve it explicitly in terms of an $N \times N$ matrix. Then, we discuss the reflection and the transmission coefficients for an arbitrary number of centres and study the threshold anomaly for the $N=2$ and $N=4$ cases. We also study further features like the quantum metastable states and resonances, including their corresponding Gamow functions and virtual or antibound states. The use of the Lippmann-Schwinger equation simplifies our analysis enormously and gives exact results for an arbitrary number of Dirac delta potentials.
\end{abstract}

Keywords: Dirac delta potentials, Lippmann-Schwinger equation, resonances, Gamow states, threshold anomaly, virtual states

(Some figures may appear in colour only in the online journal)

\section{Introduction}

As a special case of point interactions, the Dirac delta potential has been of interest for many years. A well-known application of delta potentials in physics is the Dirac comb in the Kronig Penney model, which explains band gap formation in crystal structures [1]. Another important

4 Author to whom any correspondence should be addressed. 
application of the delta potential is the description of the interaction between weakly interacting bosons through the Dirac delta functions (see e.g. chapter 15 in [2] and the references therein). The successful physical applications of the delta potentials have led to a considerable amount of work on Dirac delta potentials in different areas of physics (see [3,4] and the references therein). Among them, a remarkable study shows that scattering and reflection amplitudes of an arbitrary potential can be approximated using delta potentials [5]. More recently, the studies of several one-dimensional Dirac delta potentials based on transfer matrix techniques have been developed to illustrate some interesting scattering phenomena, namely the so-called transmission resonances (the energies for which the scattering transmission coefficient is one), threshold anomalies (for the particular values of parameters, the reflection coefficient goes to zero as the energy of the incoming particle approaches zero), Bloch states [6-11], etc.

Another important point about Dirac delta potentials is that they are exactly solvable. This also makes them useful for teaching purposes. The Green's functions and the solution of the Lippmann-Schwinger equation for a single Dirac delta potential have been given in [12] and a multiple scattering theory for double delta centres has been studied through the Lippmann-Schwinger equation in [13], from a pedagogical point of view. A recent review [3] has illustrated some other interesting features of the one-dimensional Dirac delta potentials, in particular the continuum and the bound state spectrum of the delta potentials, together with some other exactly solvable potentials. In the review, a multiple $\delta$-function potential was studied in Fourier space and the bound state problem was formulated in terms of a matrix eigenvalue problem.

In this paper, we study the details of the scattering spectrum of the multiple Dirac delta potentials through the solution of the Lippmann-Schwinger equations, which make use of the Green's functions. We give here another point of view for the treatment of the model introduced in $[6,10]$. Our point of departure is the Lippmann-Schwinger equation and we shall show how to calculate the scattering coefficients for a system of $N$ Dirac delta potentials. The method is based on the exact solution of the Lippmann-Schwinger equations, rather than solving a system of equations using the boundary conditions for delta potentials at each delta centre [10]. The method that utilizes the boundary conditions becomes rather cumbersome for $N>2$, whereas the method used here is more systematic and the results can easily be obtained for an arbitrary number of centres. Therefore, our results also suggest the use of the Lippmann-Schwinger equation for classroom exercises, including one-dimensional solvable models (e.g. multiple Dirac delta potentials), which are crucial when teaching quantum scattering.

The threshold anomaly is defined as a nonzero probability of transmission through a potential barrier, as the kinetic energy of the incident particles approaches zero [7]. Therefore, its existence is investigated by analysing the behaviour of the reflection $R(k)$ or transmission probabilities $T(k)$ for small values of $k$. It is shown in [7] that this phenomenon is related to the formation of new bound states for the given potential barrier. In this paper, we explicitly illustrate this phenomenon for two and four centres.

The contact potentials, particularly those given by two or more Dirac deltas, also serve to model quantum systems with resonances defined as quantum unstable states and/or virtual states (also called antibound states) [14-19]. The resonances can be determined from the position of the poles of the $S$-matrix in complex energy or momentum plane. In this manuscript, instead of paying attention to finding the values of the resonance poles, we focus on obtaining the Gamow functions (vectors) or eigenfunctions (eigenvectors) for the exponentially decaying part of a resonance as a direct conclusion of the Lipmann-Schwinger equation in a straightforward way. These Gamow vectors are interesting, for instance, in order to 
construct the spectral expansion for the total Hamiltonian producing quantum decaying states. We also show the appearance of the virtual states for $N=2$.

The paper is organized as follows: in section 2, starting with the Lippmann-Schwinger equation as a point of departure, we provide the solution of it in terms of an $N \times N$ matrix. The scattering information (transmission and reflection probabilities) are discussed in section 3, with particular emphasis on the simple models with one or two Dirac deltas. In section 4, we discuss the threshold anomaly for two and four Dirac delta centres. In section 5, we recall the basic definitions and features of resonances as quantum unstable states. From the Lippmann-Schwinger equation, we readily obtain the Gamow functions explicitly in this case. Finally, we discuss the appearance of the virtual (antibound) states for two centres. We also summarize the rigged Hilbert spaces and bound state formalism of the problem in appendices $\mathrm{A}$ and $\mathrm{B}$.

\section{The Lippmann-Schwinger equation for $N$ Dirac delta potentials}

Let us start with a one-dimensional model in which a free Hamiltonian of the type $H_{0}=$ $-\frac{\hbar^{2}}{2 m} \frac{\mathrm{d}^{2}}{\mathrm{~d} x^{2}}$ is perturbed with a potential $V$, so that the total Hamiltonian is $H=H_{0}+V$. In our analysis, we consider the Lippmann-Schwinger equation in momentum space. This has the advantage of simplicity, which helps very much in practical calculations as well as in its conceptual clarity. This equation can be written in the following form [20]:

$$
\left|k^{ \pm}\right\rangle=|k\rangle-G_{0}\left(E_{k} \pm \mathrm{i} 0\right) V\left|k^{ \pm}\right\rangle,
$$

where $E_{k}=k^{2} / 2 \mathrm{~m}$. The operator $G_{0}(z)=\left(H_{0}-z\right)^{-1}$ is called the Green's operator for $H_{0}$ and has interesting properties. First of all, it is an analytic function on the complex variable $z$, except at the values of the continuous spectrum of $H_{0}$, i.e. the positive semiaxis $\mathbb{R}^{+} \equiv[0, \infty)$. This means that for any vectors $\varphi, \psi$ in the Hilbert space $\mathcal{H}$, in which $H_{0}$ and $H$ act, the functions $g_{\varphi, \psi}(z)$ given by the scalar products $g_{\varphi, \psi}(z)=\left\langle\varphi \mid\left(H_{0}-z\right)^{-1} \psi\right\rangle$ are analytic on the mentioned domain denoted as $\mathbb{C} / \mathbb{R}^{+}$. Then, $G_{0}\left(E_{k} \pm \mathrm{i} 0\right)$ are the boundary values of $G_{0}(z)$ on the positive real line when we approach them from above (plus sign) or from below (minus sign). This idea is more often expressed as $\lim _{\epsilon \rightarrow 0^{+}} G_{0}\left(E_{k} \pm \mathrm{i} \epsilon\right)=G_{0}\left(E_{k} \pm \mathrm{i} 0\right)$. Since the Green's operator is discontinuous for the values of the spectrum of the free Hamiltonian-the positive semiaxis in our case-both limits are different [20].

We tacitly assume that the continuous spectrum of $H$ is also $\mathbb{R}^{+}$. The kets $|k\rangle$ and $\left|k^{ \pm}\right\rangle$are the generalized eigenvectors of $H_{0}$ and $H$, respectively, i.e. $H_{0}|k\rangle=k|k\rangle$ and $H\left|k^{ \pm}\right\rangle=k\left|k^{ \pm}\right\rangle$ for $k$ in the continuous spectrum of both operators. The signs \pm correspond to incoming and outgoing + wave functions. It is well known that these state vectors are represented by plane waves, which are not normalizable in the Hilbert space sense. Therefore, they do not belong to $\mathcal{H}$, but instead are defined as elements of a bigger structure that encompass $\mathcal{H}$, the so-called rigged Hilbert space [21-27] (see appendix A for a short overview of rigged Hilbert spaces and the continuum spectrum).

We first write the potential $V$ in the bra-ket formalism. If $|x\rangle$ are the position eigenkets with a real eigenvalue $x$, and $\psi$ is any state vector, we know that the wave function $\psi(x)$ for $\psi$ can be written as $\psi(x)=\langle x \mid \psi\rangle$. Now, for simplicity, let us assume that $V(x)=\lambda \delta(x-a)$. The action of $V$ on the wave function $\psi(x)$ is

$$
(V \psi)(x)=\lambda \delta(x-a) \psi(x)=\lambda \delta(x-a) \psi(a) .
$$

Note that $(V \psi)(x)=\langle x \mid V \psi\rangle$ and that for fixed $a$ and all $x,\langle x \mid a\rangle=\delta(x-a)$. Then, if $V=\lambda|a\rangle\langle a|$, we may write 


$$
\langle x \mid V \psi\rangle=\lambda\langle x \mid a\rangle\langle a \mid \psi\rangle=\lambda \delta(x-a) \psi(a) .
$$

Hence, we conclude that the operator 'multiplication times the Dirac delta $\delta(x-a)$ ' can be written in the bra-ket language as $|a\rangle\langle a|$.

This can be generalized to $N$ Dirac delta potentials located at different points $a_{i}$ with different strengths $\lambda_{i}$, namely

$$
V=-\sum_{i=1}^{N} \lambda_{i}\left|a_{i}\right\rangle\left\langle a_{i}\right|
$$

If we insert equation (4) into equation (1) and multiply the result from the left by the bra $\langle x|$, we obtain:

$$
\left\langle x \mid k^{ \pm}\right\rangle=\langle x \mid k\rangle+\sum_{j=1}^{N} \lambda_{i}\left\langle x\left|G_{0}\left(E_{k} \pm \mathrm{i} 0\right)\right| a_{j}\right\rangle\left\langle a_{j} \mid k^{ \pm}\right\rangle .
$$

Recall that $\langle x \mid k\rangle$ is the free plane wave, and we only keep the + sign in equation (5). Then, we find the following equation for the perturbed plane wave $\psi_{k}^{+}(x)=\left\langle x \mid k^{+}\right\rangle$as

$$
\psi_{k}^{+}(x)=\mathrm{e}^{\mathrm{i} k x}+\sum_{j=1}^{N} \lambda_{j} G_{0}\left(x, a_{j} ; E_{k}+\mathrm{i} 0\right) \psi_{k}^{+}\left(a_{j}\right)
$$

where we have used the notation $G_{0}\left(x, y ; E_{k}+\mathrm{i} 0\right)=\left\langle x\left|G_{0}\left(E_{k}+\mathrm{i} 0\right)\right| y\right\rangle$. This is the wellknown Green's function for the free Hamiltonian $H_{0}$ [20].

In order to have the explicit form for $\psi_{k}^{+}(x)$, we need to find $\psi_{k}^{+}\left(a_{j}\right)$. To do this, we just replace $x$ by $a_{i}$ in equation (6) and isolate the $j=i$ th term from the sum:

$$
\psi_{k}^{+}\left(a_{i}\right)=\mathrm{e}^{\mathrm{i} k a_{i}}+\lambda_{i} G_{0}\left(a_{i}, a_{i} ; E_{k}+\mathrm{i} 0\right) \psi_{k}^{+}\left(a_{i}\right)+\sum_{j \neq i}^{N} \lambda_{j} G_{0}\left(a_{i}, a_{j} ; E_{k}+\mathrm{i} 0\right) \psi_{k}^{+}\left(a_{j}\right),
$$

or

$$
\psi_{k}^{+}\left(a_{i}\right)\left[1-\lambda_{i} G_{0}\left(a_{i}, a_{i}: E_{k}+\mathrm{i} 0\right)\right]-\sum_{j \neq i}^{N} \lambda_{j} G_{0}\left(a_{i}, a_{j} ; E_{k}+\mathrm{i} 0\right) \psi_{k}^{+}\left(a_{j}\right)=\mathrm{e}^{\mathrm{i} k a_{i}}
$$

This relation can be written in a matrix form

$$
\sum_{j=1}^{N} \Phi_{i j}\left(E_{k}+\mathrm{i} 0\right) \psi_{k}^{+}\left(a_{j}\right)=\mathrm{e}^{\mathrm{i} k a_{i}}, \quad i=1,2, \ldots, N
$$

where the $\Phi_{i j}$ are the matrix elements of the matrix $\Phi=\left\{\Phi_{i j}\right\}$, given by

$$
\Phi_{i j}\left(E_{k}+\mathrm{i} 0\right)=\left\{\begin{array}{ccc}
1-\lambda_{i} G_{0}\left(a_{i}, a_{i}: E_{k}+\mathrm{i} 0\right) & \text { if } i=j, \\
-\lambda_{j} G_{0}\left(a_{i}, a_{j} ; E_{k}+\mathrm{i} 0\right) & \text { if } i \neq j .
\end{array}\right.
$$

The solution of equation (9) can easily be obtained as

$$
\psi_{k}^{+}\left(a_{j}\right)=\sum_{j=1}^{N}\left[\Phi^{-1}\left(E_{k}+\mathrm{i} 0\right)\right]_{j i} \mathrm{e}^{\mathrm{i} k a_{i}}
$$

where $\Phi^{-1}$ is the inverse of the matrix $\Phi$. If we substitute equation (10) in equation (6), we find 


$$
\psi_{k}^{+}(x)=\mathrm{e}^{\mathrm{i} k x}+\sum_{i, j=1}^{N} \lambda_{i} G_{0}\left(x, a_{i} ; E_{k}+\mathrm{i} 0\right)\left[\Phi^{-1}\left(E_{k}+\mathrm{i} 0\right)\right]_{i j} \mathrm{e}^{\mathrm{i} k a_{j}} .
$$

To calculate $\psi_{k}^{+}(x)$ explicitly, we first find the free Green's function $G_{0}\left(x, a_{i} ; E_{k}+\mathrm{i} 0\right)$. We insert a complete set of eigenkets of the momentum operator, $I=\int_{-\infty}^{\infty}|p\rangle\langle p| \mathrm{d} p / 2 \pi \hbar$ :

$G_{0}\left(x, a_{i} ; E_{k}+\mathrm{i} 0\right)=\left\langle x\left|G_{0}\left(E_{k}+\mathrm{i} 0\right)\right| a_{i}\right\rangle=\int_{-\infty}^{\infty}\left\langle x\left|G_{0}\left(E_{k}+\mathrm{i} 0\right)\right| p\right\rangle\left\langle p \mid a_{i}\right\rangle \frac{\mathrm{d} p}{2 \pi \hbar}$.

By definition, $|p\rangle$ are the eigenkets of the momentum operator, so that $H_{0}|p\rangle=\frac{p^{2}}{2 m}|p\rangle$. Then,

$$
\begin{aligned}
\left\langle x\left|G_{0}\left(E_{k}+\mathrm{i} 0\right)\right| p\right\rangle & =\left\langle x\left|\left(H_{0}-\left(E_{k}+\mathrm{i} 0\right)\right)^{-1}\right| p\right\rangle=\left\langle x\left|\frac{1}{\frac{p^{2}}{2 m}-\left(E_{k}+\mathrm{i} 0\right)}\right| p\right\rangle \\
& =\frac{1}{\frac{p^{2}}{2 m}-\left(E_{k}+\mathrm{i} 0\right)} \mathrm{e}^{\mathrm{i} p x / \hbar} .
\end{aligned}
$$

Using $\left\langle p \mid a_{i}\right\rangle=\mathrm{e}^{-\mathrm{i} p a_{i} / \hbar}$ and equation (14) in equation (13), we have the following expression for (13):

$$
\int_{-\infty}^{\infty} \frac{\mathrm{e}^{\mathrm{i} p\left(x-a_{i}\right) / \hbar}}{\frac{p^{2}}{2 m}-\left(E_{k}+\mathrm{i} 0\right)} \frac{\mathrm{d} p}{2 \pi \hbar}=\frac{m}{\pi \hbar} \int_{-\infty}^{\infty} \frac{\mathrm{e}^{\mathrm{i} p\left(x-a_{i}\right) / \hbar}}{p^{2}-2 m\left(E_{k}+\mathrm{i} 0\right)} \mathrm{d} p .
$$

Before we proceed to the next step, let us recall that

$$
G_{0}\left(x, a_{i} ; E_{k}+\mathrm{i} 0\right)=\lim _{\epsilon \rightarrow 0^{+}} G_{0}\left(x, a_{i} ; E_{k}+\mathrm{i} \epsilon\right) .
$$

This remark is essential in order to understand the meaning of the integral in (15), which is nothing else but

$$
\int_{-\infty}^{\infty} \frac{\mathrm{e}^{\mathrm{i} p\left(x-a_{i}\right) / \hbar}}{p^{2}-2 m\left(E_{k}+\mathrm{i} 0\right)} \mathrm{d} p=\lim _{\epsilon \rightarrow 0^{+}} \int_{-\infty}^{\infty} \frac{\mathrm{e}^{\mathrm{i} p\left(x-a_{i}\right)}}{p^{2}-2 m\left(E_{k}+\mathrm{i} \epsilon\right)} \mathrm{d} p .
$$

It is very important to understand that the limit and the integral in (17) cannot be interchanged, since it is the right-hand side of (17) that gives sense to the left-hand side. In fact, we have to solve the integral first and then proceed with the limit. Let us solve the integral in the right-hand side of (17) by the residue method. The function under the integral sign has poles at the points

$$
p= \pm \hbar k \sqrt{1+\mathrm{i} \frac{2 m \epsilon}{\hbar^{2} k^{2}}} \simeq \pm\left(\hbar k+\mathrm{i} \frac{m \epsilon}{\hbar k}\right),
$$

where $E_{k}=\hbar^{2} k^{2} / 2 m$ and $k>0$. In order to calculate the integral by the residue method, we have to take into account that either $x<a_{i}$ or $x>a_{i}$. In the second case, we use the contour depicted in the left contour of figure 1 .

We note that only the pole with the plus sign in equation (18) lies inside the contour of integration. The integral over the semicircle vanishes as its radius goes to infinity [28]. Then, the value of the integral is obtained, multiplying the residue at that point by $2 \pi \mathrm{i}$. This gives

$$
\mathrm{i} \frac{m}{\hbar}\left[\lim _{\epsilon \rightarrow 0^{+}} \frac{\mathrm{e}^{\mathrm{i}\left(k+\frac{m \epsilon}{k}\right)\left(x-a_{i}\right)}}{\hbar k+\mathrm{i} \frac{m \epsilon}{\hbar k}}\right]=\mathrm{i} m \frac{\mathrm{e}^{\mathrm{i} k\left(x-a_{i}\right)}}{\hbar^{2} k} .
$$

This is the result of the integral (17) for $x>a_{i}$. For $x<a_{i}$ we use a similar contour defined in the lower half plane, as shown in the right contour of figure 1 . Then, the relevant pole is the 

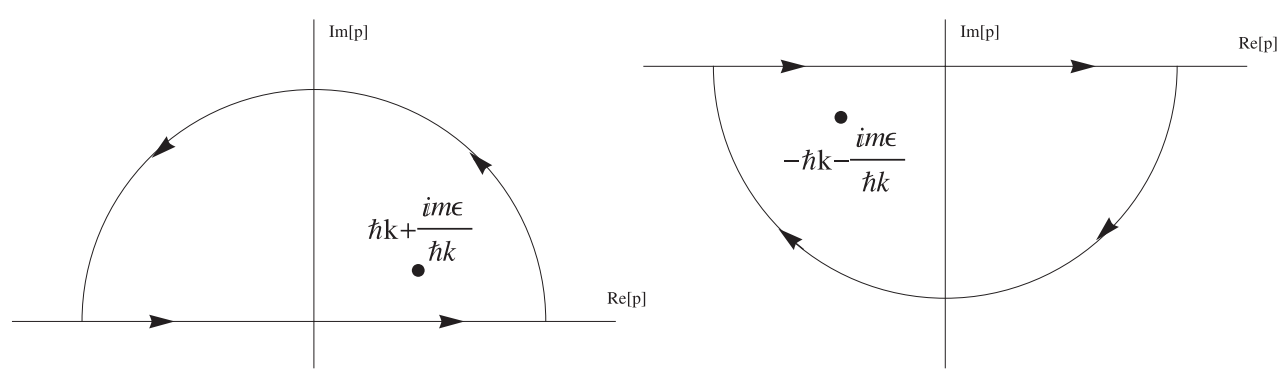

Figure 1. We choose the left contour for the residue calculation for $x>a_{i}$ and the right one for $x<a_{i}$.

one with the minus sign in equation (18). This gives a similar result as in (19), replacing the $k$ in the exponential with $-k$. Therefore, for all values of $x$ we can write

$$
G_{0}\left(x, a_{i} ; E_{k}+\mathrm{i} 0\right)=\int_{-\infty}^{\infty} \frac{\mathrm{e}^{\mathrm{i} p\left(x-a_{i}\right) / \hbar}}{\frac{p^{2}}{2 m}-\left(E_{k}+\mathrm{i} 0\right)} \frac{\mathrm{d} p}{2 \pi \hbar}=\frac{\mathrm{i} m}{\hbar^{2} k} \mathrm{e}^{k\left|x-a_{i}\right|}
$$

Substituting this result into formula (12), we finally obtain

$$
\psi_{k}^{+}(x)=\mathrm{e}^{\mathrm{i} k x}+\sum_{i=1}^{N} \sum_{j=1}^{N} \frac{\mathrm{i} m \sqrt{\lambda_{i} \lambda_{j}}}{\hbar^{2} k} \mathrm{e}^{\mathrm{i} k\left|x-a_{i}\right|}\left[\Phi^{-1}\left(E_{k}+\mathrm{i} 0\right)\right]_{i j} \mathrm{e}^{\mathrm{i} k a_{j}},
$$

where

$$
\Phi_{i j}\left(E_{k}+\mathrm{i} 0\right)=\left\{\begin{array}{cl}
1-\frac{\mathrm{i} m \lambda_{i}}{\hbar^{2} k} & \text { if } i=j, \\
-\sqrt{\lambda_{i} \lambda_{j}} \frac{\mathrm{i} m}{\hbar^{2} k} \mathrm{e}^{\mathrm{i} k\left|a_{i}-a_{j}\right|} & \text { if } i \neq j .
\end{array}\right.
$$

Note that $\Phi^{-1}(E+\mathrm{i} 0)$ is the inverse of the matrix with the elements given above equation (22).

\section{The reflection and the transmission coefficients}

We assume that the incoming particles come from the left. Then, from the coefficient of $\mathrm{e}^{-\mathrm{i} k x}$ in the solution $\psi_{k}^{+}(x)$ when $x<a_{i}$, we can find the reflection amplitude

$$
r(k)=\sum_{i, j=1}^{N} \frac{\mathrm{i} m \sqrt{\lambda_{i} \lambda_{j}}}{\hbar^{2} k} \mathrm{e}^{\mathrm{i} k a_{i}}\left[\Phi^{-1}\left(E_{k}+\mathrm{i} 0\right)\right]_{i j} \mathrm{e}^{\mathrm{i} k a_{j},}
$$

and from the coefficient of $\mathrm{e}^{\mathrm{i} k x}$ in the solution $\psi_{k}^{+}(x)$ for $x>a_{i}$, we can find the transmission amplitude

$$
t(k)=1+\sum_{i, j=1}^{N} \frac{\mathrm{i} m \sqrt{\lambda_{i} \lambda_{j}}}{\hbar^{2} k} \mathrm{e}^{-\mathrm{i} k a_{i}}\left[\Phi^{-1}\left(E_{k}+\mathrm{i} 0\right)\right]_{i j} \mathrm{e}^{\mathrm{i} k a_{j}} .
$$

It is instructive to show that the solution $\psi_{k}^{+}(x)$ and the reflection and the transmission amplitudes given above yield the standard textbook results if we consider one Dirac delta potential located at $x=a$ with strength $\lambda$. 
For $N=1$, the matrix $\Phi$ is simply a function, namely

$$
\Phi_{11}\left(E_{k}+\mathrm{i} 0\right)=1-\frac{\mathrm{i} m \lambda}{\hbar^{2} k},
$$

where we have chosen $\lambda_{1}=\lambda$ and used $\lim _{\epsilon \rightarrow 0^{+}} \sqrt{-2 m\left(E_{k}+\mathrm{i} \epsilon\right)}=-\mathrm{i} \hbar k$. Then, the above formula (21) is reduced to

$$
\psi_{k}^{+}(x)= \begin{cases}e^{\mathrm{i} k x}+\frac{\mathrm{i} m \lambda}{\hbar^{2} k} \frac{e^{-\mathrm{i} k(x-a)}}{1-\mathrm{i} \frac{m \lambda}{\hbar^{2} k}} & \text { if } x<a, \\ e^{\mathrm{i} k x}+\frac{\mathrm{i} m \lambda}{\hbar^{2} k} \frac{e^{\mathrm{i} k(x-a)}}{1-\mathrm{i} \frac{m \lambda}{\hbar^{2} k}} & \text { if } x>a .\end{cases}
$$

Substituting the function $\Phi$ given in equation (25) into equations (23) and (24), we obtain the following well-known textbook result [29]:

$$
R(k)=|r(k)|^{2}=\frac{m^{2} \lambda^{2}}{\hbar^{4} k^{2}+m^{2} \lambda^{2}}, \quad T(k)=|t(k)|^{2}=\frac{\hbar^{4} k^{2}}{\hbar^{4} k^{2}+m^{2} \lambda^{2}} .
$$

Note that $R+T=1$. Moreover, $T \mapsto 1$ as $k \mapsto \infty$ as expected.

In order to compare our results with those given in [10], let us choose $N=2$. Then, matrix $\Phi$ has the following form:

$$
\Phi=\left(\begin{array}{cc}
1-\frac{\mathrm{i} m \lambda}{\hbar^{2} k} & -\frac{\mathrm{i} m \lambda}{\hbar^{2} k} e^{\mathrm{i} \mathrm{k}\left|a_{1}-a_{2}\right|} \\
-\frac{\mathrm{i} m \lambda}{\hbar^{2} k} e^{\mathrm{ik}\left|a_{1}-a_{2}\right|} & 1-\frac{\mathrm{i} m \lambda}{\hbar^{2} k}
\end{array}\right) .
$$

For a better comparison with [10], let us choose $a_{1}=-a$ and $a_{2}=a$ with $a>0$. Then,

$$
\Phi^{-1}=\frac{1}{\operatorname{det} \Phi}\left(\begin{array}{ll}
1-\frac{\mathrm{i} m \lambda}{\hbar^{2} k} & \frac{\mathrm{i} m \lambda}{\hbar^{2} k} e^{\mathrm{i} 2 k a} \\
\frac{\mathrm{i} m \lambda}{\hbar^{2} k} e^{\mathrm{i} 2 k a} & 1-\frac{\mathrm{i} m \lambda}{\hbar^{2} k}
\end{array}\right)
$$

with

$$
\operatorname{det} \Phi=1-\frac{2 \mathrm{i} m \lambda}{\hbar^{2} k}+\frac{m^{2} \lambda^{2}}{\hbar^{4} k^{2}}\left(e^{4 \mathrm{i} k a}-1\right) .
$$

These formulae are necessary for constructing $\psi_{k}^{+}(x)$ as in equation (12) for $N=2$. All scattering features can be obtained through this wave function. In particular, we may obtain the reflection and transmission coefficients. The wave function (12) reads for $x<-a$ :

$$
\begin{aligned}
\psi_{k}^{+}(x)= & e^{\mathrm{i} k x}+\frac{\mathrm{i} m \lambda}{\hbar^{2} k} e^{-\mathrm{i} k(x+a)} \Phi_{11}^{-1} e^{-\mathrm{i} k a}+\frac{\mathrm{i} m \lambda}{\hbar^{2} k} e^{-\mathrm{i} k(x+a)} \Phi_{12}^{-1} e^{\mathrm{i} k a} \\
& +\frac{\mathrm{i} m \lambda}{\hbar^{2} k} e^{-\mathrm{i} k(x-a)} \Phi_{21}^{-1} e^{-\mathrm{i} k a}+\frac{\mathrm{i} m \lambda}{\hbar^{2} k} e^{-\mathrm{i} k(x-a)} \Phi_{22}^{-1} e^{\mathrm{i} k a} .
\end{aligned}
$$

Then, from equation (23), we explicitly find the reflection amplitude

$$
r(k)=(\operatorname{det} \Phi)^{-1} \frac{\mathrm{i} m \lambda}{\hbar^{2} k}\left[\left(1-\frac{\mathrm{i} m \lambda}{\hbar^{2} k}\right)\left[\mathrm{e}^{-2 \mathrm{i} k a}+\mathrm{e}^{2 \mathrm{i} k a}\right]+2\left(\frac{\mathrm{i} m \lambda}{\hbar^{2} k}\right) \mathrm{e}^{2 \mathrm{i} k a}\right] .
$$

The values of $k$ for which $T(k)=1$ are known as transmission resonances in the literature [8]. One can plot the graph of $T$ as a function of $k$ and read the transmission resonances, as shown in the top right part of figure 2 for $N=2$. To find these values explicitly, we can equivalently find the roots of the transcendental equation $r(k)=0$ numerically. It has been shown in [10] 

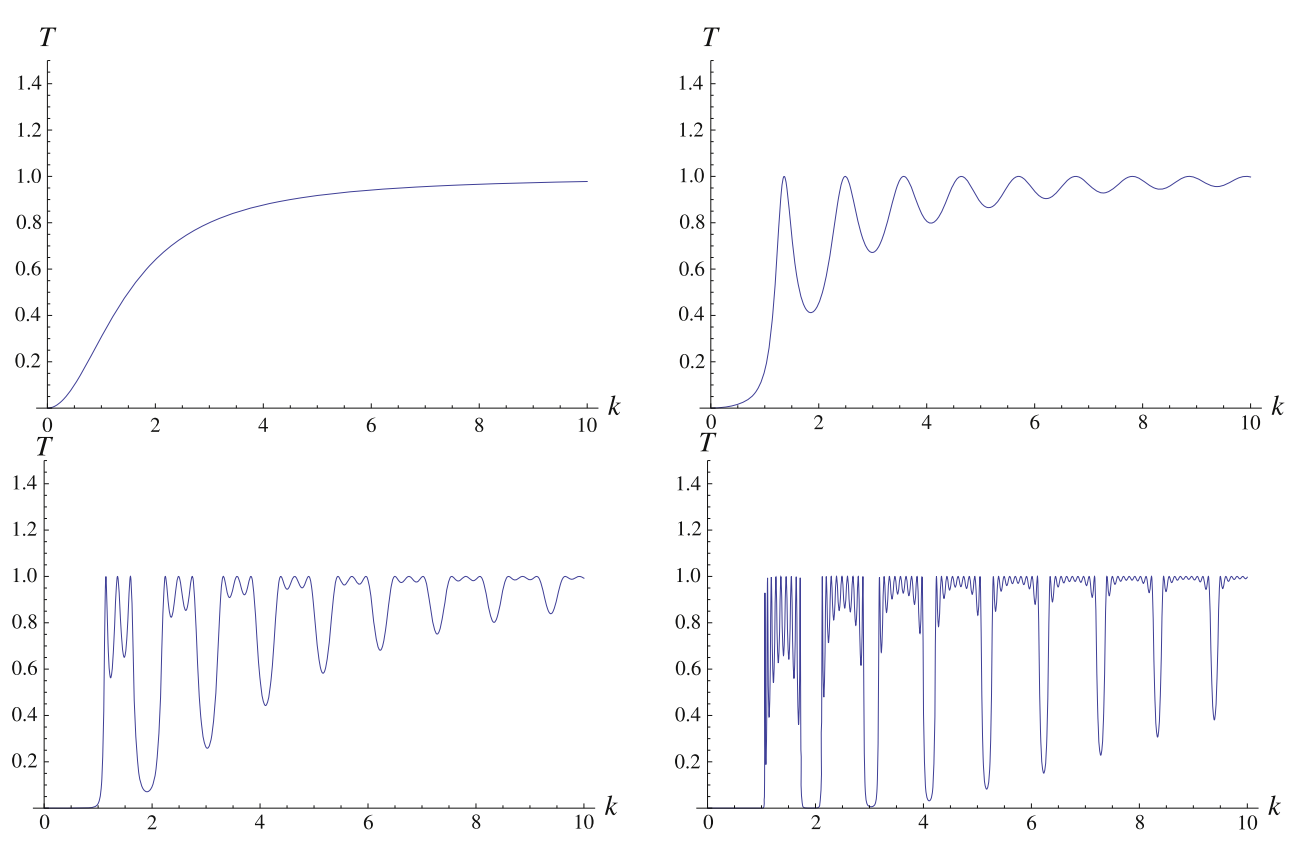

Figure 2. The transmission coefficient as a function of $k$ for $N=1,2,4,10$, respectively. Here, $\lambda_{i}=2$ and $\left|a_{i}-a_{j}\right|=3$ for all $i, j$. We use units such that $\hbar=2 m=1$.

that the energies corresponding to the transmission resonances are different from the resonance energies obtained from the poles of the $S$-matrix or transmission coefficient.

The transmission coefficient for the Dirac delta potentials located periodically in the positive real axis is depicted for different numbers of $N$ in figure 2. This illustrates the appearance of band gaps as we increase the number of centres, which was first shown in [30].

\section{Threshold anomaly}

The threshold anomaly occurs for potentials consisting of $N(\geqslant 2)$ Dirac delta potentials. We start to analyse the threshold anomalies for two Dirac delta centres by investigating $R(k)$ for small values of $k$. To this end, we expand it around $k=0$ :

$$
R(k)=\left|-1+\frac{\mathrm{i}\left(-1+\frac{8 a^{2} m^{2} \lambda^{2}}{\hbar^{4}}\right)}{2 m \lambda\left(-1+\frac{2 m a \lambda}{\hbar^{2}}\right)} \hbar^{2} k+O\left(k^{2}\right)\right|^{2} .
$$

It is easy to see that $R(k) \rightarrow 1$ as $k \rightarrow 0$, unless $\frac{2 m a \lambda}{\hbar^{2}}=1$. This means that the reflection probability gets closer and closer to one as the energy of the incoming particles decreases, except for the critical case $\frac{2 m a \lambda}{\hbar^{2}}=1$. This is the generic case, and can be seen from figure 2 . To understand the behaviour of $R$ near $k=0$ in the critical case, we first substitute $a=\frac{\hbar^{2}}{2 m \lambda}$ into $R(k)(r(k)$ is given in equation (32)), and then expand it near $k=0$ so that 


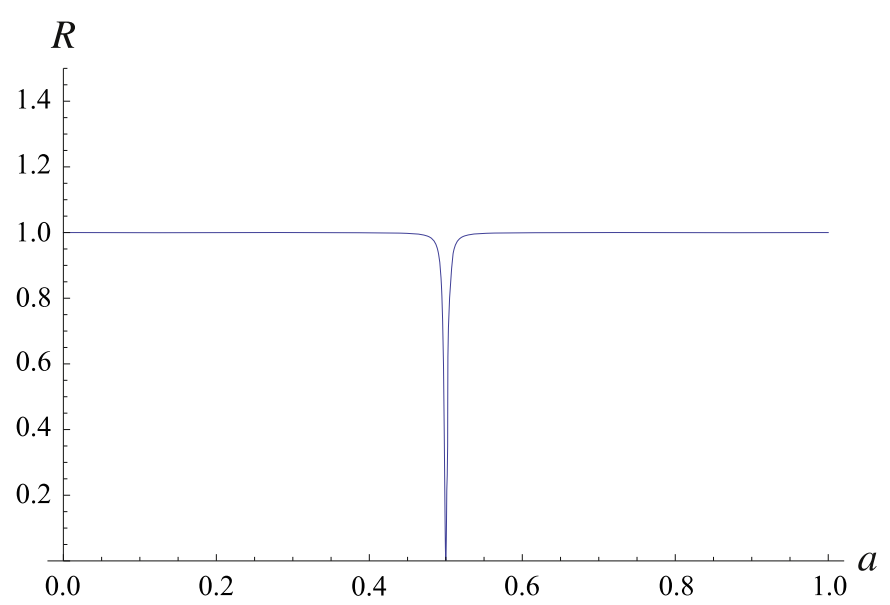

Figure 3. The reflection coefficient as a function of $a$ for two centres. Here, $\lambda_{1}=\lambda_{2}=2$ and $k=0.01(\hbar=2 m=1)$.

$$
R(k)=\left|\frac{4 \mathrm{i} a}{3} k+\frac{32 a^{2}}{9} k^{2}+O\left(k^{3}\right)\right|^{2}
$$

This shows that the probability of reflection of the particle vanishes as the kinetic energy of the incoming particles goes to zero. This phenomenon is known as the threshold anomaly and was first discussed in [7]. Actually, this fact can be seen more transparently by plotting the reflection coefficient as a function of $a$ near $k=0$.

As can be seen from figure 3 , the reflection coefficient sharply drops to zero at exactly the same critical value $a=\hbar^{2} / 2 m \lambda$. This is due to the appearance of a second bound state at this critical value. Note that this is exactly the same condition for the formation of the second bound state, as discussed in our recent paper [31] (the only difference is that the centres are separated by $a$ in the paper). In other words, the underlying reason for this is the appearance of a bound state very close to the threshold energy [7].

For the $N=4$ case, the reflection coefficient near $k=0$ is

$$
R(k)=\left|-1+\frac{\mathrm{i}\left(384 a^{4} m^{4} \lambda^{4}-512 a^{3} m^{3} \lambda^{3} \hbar^{2}+160 a^{2} m^{2} \lambda^{2} \hbar^{4}-\hbar^{8}\right)}{4 m \lambda\left(2 a m \lambda-\hbar^{2}\right)\left(8 a^{2} m^{2} \lambda^{2}-8 a m \lambda \hbar^{2}+\hbar^{4}\right)} k+O\left(k^{2}\right)\right|^{2} .
$$

Similar to the $N=2$ case, $R(k) \rightarrow 1$ as $k \rightarrow 0$, unless the denominator is zero, that is,

$$
\begin{aligned}
2 a m \lambda-\hbar^{2} & =0 \\
\left(8 a^{2} m^{2} \lambda^{2}-8 a m \lambda \hbar^{2}+\hbar^{4}\right) & =0 .
\end{aligned}
$$

The solution to these equations is

$$
m \lambda a=\left\{\frac{\hbar^{2}}{4}(2-\sqrt{2}), \frac{\hbar^{2}}{2}, \frac{\hbar^{2}}{4}(2+\sqrt{2})\right\} .
$$




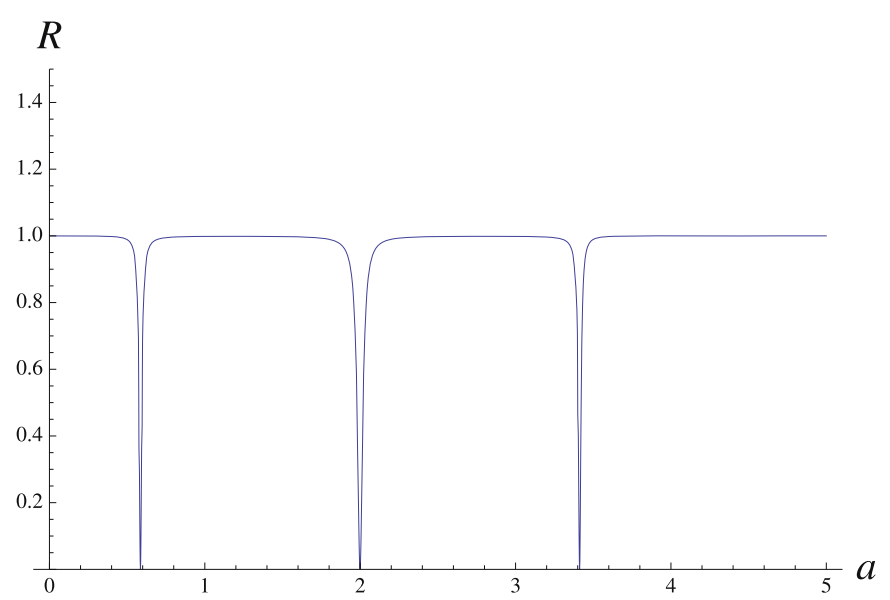

Figure 4. The reflection coefficient as a function of $a$ for four centres. Here, $\lambda_{1}=\lambda_{2}=1 / 2$ and $k=0.01(\hbar=2 m=1)$.

If we first substitute these solutions into $R(k)$ and then expand it, we obtain

$$
R(k)=\left\{\begin{array}{cc}
\left|\frac{8 \mathrm{i} a k}{3}+O\left(k^{2}\right)\right|^{2} & \text { when } m \lambda a=\frac{\hbar^{2}}{2}, \\
\left|-\frac{4}{3} \mathrm{i}(-3 a+\sqrt{2} a) k+O\left(k^{2}\right)\right|^{2} & \text { when } m \lambda a=\frac{\hbar^{2}}{4}(2-\sqrt{2}), \\
\left|\frac{4}{3} \mathrm{i}(3 a+\sqrt{2} a) k+O\left(k^{2}\right)\right|^{2} & \text { when } m \lambda a=\frac{\hbar^{2}}{4}(2+\sqrt{2}) .
\end{array}\right.
$$

This shows the threshold anomaly for the $N=4$ case. One can also see from figure 4 the vanishing behaviour of the reflection coefficient at the above critical cases near $k=0$. All those critical values of $a$ for which $R$ vanishes near $k=0$ are just the critical values of the parameters in the model where the new bound states appear. In order to show this, we plot the flow of eigenvalues of the principal matrix (see equation (B-2) in appendix B) as a function of $|E|$, as shown in figure 5 by fixing the value of the parameter $a=1$. Figure 5 shows the flow of the eigenvalues of the matrix $\Phi$, where the zeros of the eigenvalues are the bound state energies. The top left graph shows the critical situation $m \lambda a=\frac{\hbar^{2}}{4}(2-\sqrt{2})$, where the second bound state appears. Similarly, the other two graphs show the critical cases $m \lambda a=\frac{\hbar^{2}}{4}(2+\sqrt{2})$ and $m \lambda a=\frac{\hbar^{2}}{2}$, respectively. It is easy to notice that all the critical values for the appearance of the new bound states correspond exactly to the values of the parameter where the threshold anomaly occurs.

All these show that the method that we have used is very useful and systematic for large values of centres, whereas the standard method may become very cumbersome in dealing with more than two.

\section{Resonances and Gamow states}

There are basically two legitimate approaches to quantum resonances. In the first one, one considers the decaying system as an open system, that is, it interacts with its environment. In the second one, although the system is closed (isolated) and the Hamiltonian is self adjoint, 


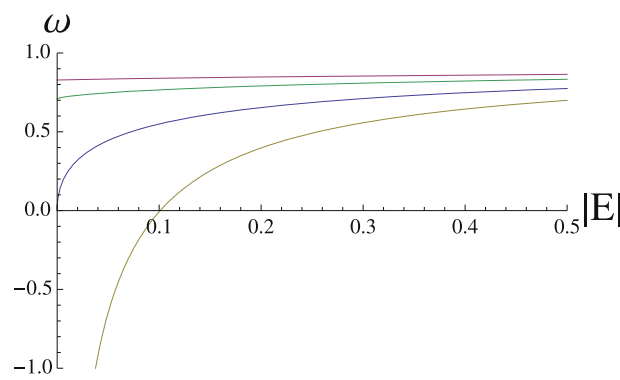

(a) $\lambda_{c}=\frac{1}{2}(2-\sqrt{2}), E_{1}=-0.101$

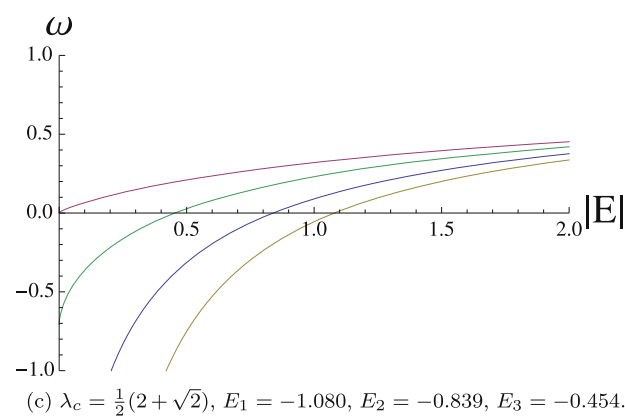

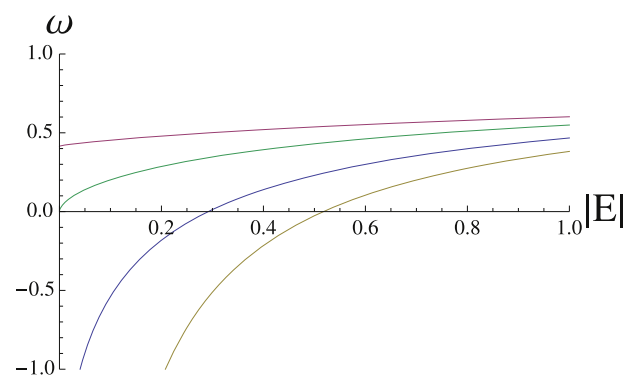

(b) $\lambda_{c}=1, E_{1}=-0.518, E_{2}=-0.292$.

Figure 5. The flow of the eigenvalues of the principal matrix $\Phi$ for the critical values of the parameter $\lambda_{c}(\hbar=2 m=1)$ and the bound state energies. We choose $a=1$ in all cases.

we may still have resonances and there exist Gamow vectors with complex eigenvalues. The point is to extend the Hamiltonian by the duality to a bigger space containing non-normalizable eigenfunctions such as the Gamow states; see [38] for the details.

Models with two Dirac delta potentials in the context of resonances have been discussed, for instance, in [14, 17], and see references therein. We recall that the resonances are always produced by a perturbation on the free Hamiltonian. In other words, in order to have resonances, we need a Hamiltonian pair $\left(H_{0}, H\right)$, where $H_{0}$ is 'free' or unperturbed and $H=H_{0}+V$ is the total Hamiltonian. In our case, the perturbation is given by a multiple Dirac delta potential $V$.

Let us emphasize a very important point about one-dimensional scattering, which has been previously mentioned. Concerning resonances and perfect transmission values, a certain confusion arises in the literature, see for example [6]. For some types of one-dimensional potential there exist values of energy for which the transmission coefficient is equal to one. They are sometimes called resonances or transmission resonances. In [10], the terminology perfect transmission is proposed to describe this phenomenon, but we will stick to transmission resonances. On the other hand, we shall reserve the name resonance for a type with poles of the scattering $S$-matrix. If the $S$-matrix is written in terms of its dependence on the momentum $k$ of the incident particle, $S(k)$, and satisfies some general assumptions, then it admits an analytic continuation to the complex values of $k$. Then, the resonances are characterized by pairs of poles of $S(k)$ located in the lower half plane symmetrically with respect to the imaginary axis. These pairs of poles reveal the existence of quantum unstable states or resonances, and both terms denote the same physical reality. One can find a deep and thorough study of this in $[32,33]$. 
Nevertheless, the poles of the transmission coefficient coincide with the poles of the scattering $S$-matrix [34]. Due to this fact, resonance poles can be calculated by using the transcendental equation $\operatorname{det} \Phi=0$. We may find four types of solution:

1. Simple poles in the positive imaginary $k$ axis which have the form i $\alpha$, with $\alpha>0$ and are associated with the bound states [33].

2. Simple poles in the negative imaginary $k$ axis which have the form $-\mathrm{i} \alpha$, with $\alpha>0$ and whose physical meaning is not always clear [35-37]. They are associated with the states known as antibound or virtual [32, 33].

3. A possible pole at the origin. This may be associated with the situation in which causality conditions, as usually stated, do not hold [32].

4. Pairs of poles of the form $\pm k_{0}-\mathrm{i} k_{1}$ with $k_{0}, k_{1}>0$. Each pair of poles of this kind represents a resonance $[32,33]$. In principle, these poles may have any (finite) multiplicity, although for realistic models this multiplicity is one. The number of resonance pairs of poles in systems with a finite range of potentials (the potential is zero outside a bounded region) is infinite.

The method used in [10] is based on the usage of the continuity and jump conditions of the wave function and its derivative at the location of the Dirac delta centres, respectively. In contrast, we adopt the Lippmann-Schwinger equation to find the scattering and resonance information for the system. When $N=2$, the Hamiltonian in [10] is given as $H=$ $-\frac{\hbar^{2}}{2 m} \frac{\mathrm{d}^{2}}{\mathrm{~d} x^{2}}+V_{1} \delta(x)+V_{2} \delta(x-a)$. Since the Hamiltonian that we consider is $H=-$ $\frac{\hbar^{2}}{2 m} \frac{\mathrm{d}^{2}}{\mathrm{~d} x^{2}}-\lambda \delta(x-a)-\lambda \delta(x+a)$, one can easily check that equation (28) in [10], namely $\left(2 \mathrm{i} k-\nu_{1}\right)\left(2 \mathrm{i} k-\nu_{2}\right)=\nu_{1} \nu_{2} \mathrm{e}^{2 \mathrm{i} k a}$, where $\nu_{i}=\frac{2 m V_{i}}{\hbar^{2}}$ is identical to our equation $\operatorname{det} \Phi=0$ (det $\Phi$ is given by (30). This can be seen by identifying $V_{1}$ and $V_{2}$ with $-\lambda$ and relabelling $a \rightarrow 2 a$ in equation (28) in [10]. Therefore, we conclude that the results in [10] for the scattering states and resonances are consistent with our results. Note that in [10], the locations of the resonance poles are given in terms of the complex energies. These complex energies are calculated using the formula $E=\hbar^{2} k^{2} / 2 m$, so that if $k= \pm k_{0}-\mathrm{i} k_{1}$, then $E \pm \mathrm{i} \hbar \Gamma / 2=\left(\mp k_{0}-\mathrm{i} k_{1}\right)^{2}$. The real part $E$ is called the resonant energy and the imaginary part is related to the mean life of the resonance [33].

At this point, we need to remark that the transformation $E:=\hbar^{2} k^{2} / 2 m$ transforms the function $S(k)$ into a function $S(E)$, which is now defined in a two-sheeted Riemann surface, where each sheet is a complex plane, see [33]. In this case, the resonance poles are complex conjugate poles located on the second sheet at the points $E \pm \mathrm{i} \hbar \Gamma / 2$, with $E, \Gamma>0$. Both complex conjugate poles represent the same resonance.

By definition, a Gamow state $\psi$ is an eigenvector (wave function) of the total Hamiltonian $H=H_{0}+V$ having the complex eigenvalue, $H \psi=(E-\mathrm{i} \Gamma / 2) \psi$, where $E-\mathrm{i} \Gamma / 2$ is called the resonance pole. Then, for the Gamow wave functions $\psi$ we have

$$
\mathrm{e}^{-\frac{\mathrm{i}}{\hbar} t H} \psi=\mathrm{e}^{-\frac{\mathrm{i}}{\hbar} E t} \mathrm{e}^{-\Gamma t / 2} \psi
$$

so that $\psi$ decays exponentially with time. For this reason, Gamow wave functions may be seen as state wave functions for the resonances.

However, we have two problems in this interpretation. The former has to do with the selfadjointness of the Hamiltonian $H$. A self-adjoint Hamiltonian cannot have complex eigenvalues in a Hilbert space. The only possible solution is to extend the Hilbert space to a larger space which does not have normalizable wave functions so that equation $H \psi=$ $(E-\mathrm{i} \hbar \Gamma / 2) \psi$ as well as equation (39) make sense in this larger space. This has been done with the help of the rigged Hilbert spaces-a mathematical tool that was initially used to 
introduce a rigorous presentation of the Dirac formalism for quantum mechanics [21, 23-26]. Note that we represent the resonance by one of the resonance poles $E-\mathrm{i} \Gamma / 2$. The other one $(E+\mathrm{i} \hbar \Gamma / 2)$ will play a symmetric role which will go beyond the scope of the present paper.

The second difficulty comes from the fact that it is not clear that the quantum decaying systems decay exponentially for all times. In any case, experiments have shown that exponential decay is a very good approximation for decay behaviour in essentially all ranges of time, with the possible exception of very short or very long times. Thus, the Gamow wave functions can be good approximations of the wave functions of decaying states for the majority values of time. In our case, being given a resonance defined by a pair of poles of the $S$-matrix, we may construct its Gamow wave function. The method is to use an analytic continuation of equation (2) and we shall use a description of it. The technicalities can be found in [38,39]. Let $E_{R}-\mathrm{i} \hbar \Gamma / 2$ be the location of a resonance pole in the energy representation. The analytic continuation of (2) at the complex value $k_{R}$ so that $z_{R}:=E_{R}-\mathrm{i} \hbar \Gamma / 2=k_{R}^{2} \hbar^{2} / 2 m$ is given by

$$
\left|k_{R}^{+}\right\rangle=\left|k_{R}\right\rangle-G_{0}\left(z_{R}\right) V\left|k_{R}^{+}\right\rangle
$$

where $\left|k_{R}^{+}\right\rangle$and $z_{R}$ are the eigenvectors and eigenvalues of $H$, respectively:

$$
H\left|k_{R}^{+}\right\rangle=\frac{k_{R}^{2} \hbar^{2}}{2 m}\left|k_{R}^{+}\right\rangle=z_{R}\left|k_{R}^{+}\right\rangle .
$$

Thus, $\left|k_{R}^{+}\right\rangle$is the Gamow vector for the resonance with the resonance pole $k_{R}$. This Gamow vector in the coordinate representation is $\psi_{R}(x):=\left\langle x \mid k_{R}^{+}\right\rangle$, so that

$$
\left(H \psi_{R}\right)(x)=\left\langle x|H| k_{R}^{+}\right\rangle=z_{R}\left\langle x \mid k_{R}^{+}\right\rangle=z_{R} \psi_{R}(x) .
$$

If we use $V=-\sum_{i=1}^{N} \lambda_{i}\left|a_{i}\right\rangle\left\langle a_{i}\right|$ and multiply (40) from the left by $\langle x|$, we obtain

$$
\left\langle x \mid k_{R}^{+}\right\rangle=\left\langle x \mid k_{R}\right\rangle+\sum_{i=1}^{N} \lambda_{i}\left\langle x\left|G_{0}\left(z_{R}\right)\right| a_{i}\right\rangle\left\langle a_{i} \mid k_{R}^{+}\right\rangle .
$$

Let us write the complex number $k_{R}$ in terms of its real and imaginary parts as $k_{R}=k_{r}-\mathrm{i} k_{I}$. Since $\left\langle x \mid k_{R}\right\rangle=\mathrm{e}^{\mathrm{i} k_{R} x}$ by the process of analytic continuation, equation (43) becomes

$$
\psi_{R}^{+}(x)=\mathrm{e}^{\mathrm{i} k_{r} x} \mathrm{e}^{k_{l} x}+\sum_{i=1}^{N} \lambda_{i} G_{0}\left(x, a_{i} ; z_{R}\right) \psi_{R}^{+}\left(a_{i}\right) .
$$

Proceeding as in section 2, we can find $\psi_{R}^{+}\left(a_{i}\right)$ so that

$\psi_{R}^{+}(x)=\mathrm{e}^{\mathrm{i} k_{r} x} \mathrm{e}^{k_{I} x}+\sum_{i=1}^{N} \sum_{j=1}^{N} \frac{\mathrm{i} m \sqrt{\lambda_{i} \lambda_{j}}}{\hbar^{2}\left(k_{r}+\mathrm{i} k_{I}\right)} \mathrm{e}^{\mathrm{i}\left(k_{r}+\mathrm{i} k_{l}\right)\left|x-a_{i}\right|}\left[\Phi^{-1}\left(z_{R}\right)\right]_{i j} \mathrm{e}^{\mathrm{i}\left(k_{r}+\mathrm{i} k_{l}\right) a_{j}}$.

We now observe that the first term in the right-hand side of (44) diverges exponentially as $x \longmapsto \infty$. In the second term, we have a sum including the Green's function for the free Hamiltonian $H_{0}$, given by

$$
\begin{aligned}
G_{0}\left(x, a_{j} ; z_{R}\right) & =\frac{\mathrm{i} m}{\hbar} \frac{\exp \left\{\frac{\mathrm{i}}{\hbar} \sqrt{2 m z_{R}}\left|x-a_{j}\right|\right\}}{\sqrt{2 m z_{R}}} \\
& =\frac{\mathrm{i} m}{\hbar} \frac{\exp \left\{\frac{\mathrm{i}}{\hbar} \sqrt{2 m} k_{r}\left|x-a_{j}\right|\right\} \exp \left\{\frac{1}{\hbar} \sqrt{2 m} k_{I}\left|x-a_{j}\right|\right\}}{\sqrt{2 m z_{R}}} .
\end{aligned}
$$

Again since $k_{I}$ is positive, we observe exponential behaviour for large values of $|x|$. The conclusion is that Gamow wave functions cannot be normalized in the sense of square 

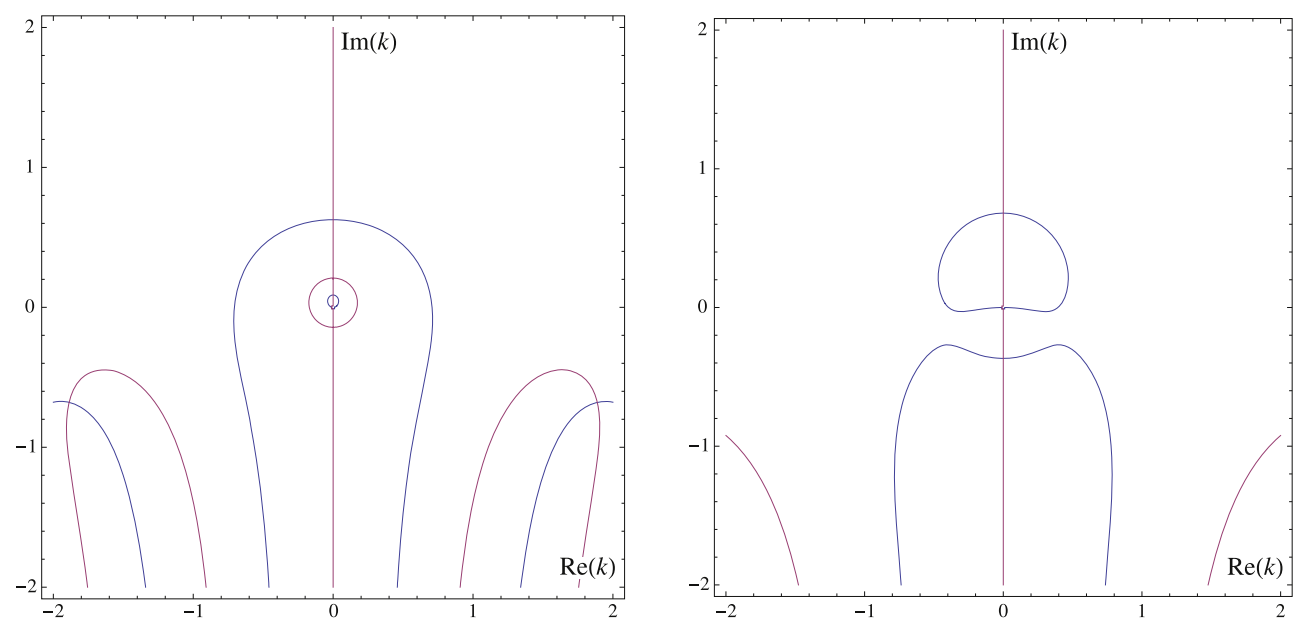

Figure 6. Contour plots of the $\mathfrak{R}(\operatorname{det} \Phi)=0$ and $\mathfrak{I}(\operatorname{det} \Phi)=0$ for $a=2.2$ on the left and $a=1.5$ on the right; $\lambda_{1}=\lambda_{2}=1$ in both cases.

integrability, but in contrast with Dirac kets or plane waves, which are bounded but not square integrable, they show exponential behaviour at infinity in the coordinate representation. This behaviour is sometimes called the spatial or exponential catastrophe. With a proper interpretation of the Gamow wave functions in terms of generalized functions in the rigged Hilbert spaces, one may show that this is far from being a catastrophe [39].

\section{Virtual states for $N=2$}

In this short section, we summarize the results on how the location of the poles of the transmission coefficient (or $S$-matrix) changes with respect to the distance between two attractive Dirac delta potentials within our formalism. As previously emphasized, the poles of the transmission coefficients are given by the solution of the transcendental equation $\operatorname{det} \Phi(k)=0$.

Here we choose that $a_{1}=0$ and $a_{2}=a$, and the units such that $\hbar=2 \mathrm{~m}=1$. Then, the complex solutions of $\operatorname{det} \Phi\left(E_{k}+\mathrm{i} 0\right)=0$ can be found in the lower half plane of the complex $k$ plane. In order to follow the motion of these poles, we plot the zero level curves of $\mathfrak{R}\left(\operatorname{det} \Phi\left(E_{k}+\mathrm{i} 0\right)\right)$ (blue curves in figure 6) and $\mathfrak{I}\left(\operatorname{det} \Phi\left(E_{k}+\mathrm{i} 0\right)\right)$ (red curves in figure 6) by fixing $\lambda$ for different values of $a$. The intersections of the red and blue curves on the complex $k$ plane are the solutions of $\operatorname{det} \Phi\left(E_{k}+\mathrm{i} 0\right)=0$. The simple poles on the positive imaginary $k$ axis correspond to the bound states, whereas the ones in the negative imaginary $k$ axis are known as virtual states [20]. As emphasized in [31], it is well known that there are two bound states when $a$ is sufficiently large $\left(a>\hbar^{2} / m \lambda\right.$ or the critical case $a=2$ for a particular choice of the parameter $\lambda=2$ ) and the second bound state eventually becomes a virtual state as we decrease the distance between the centres. From figure 6, if the distance between the centres is $d=2 a=1.4$, the second bound state pole is shifted to the negative imaginary axis. Thus, it becomes a virtual state. This phenomenon also occurs for some other potentials as well, e.g. a rectangular well and barrier potentials [40, 41]. Although $k=0$ seems to be a solution from figure 6 , it is a singular point of $\operatorname{det} \Phi$ given in equation (30). This is due to the lack of resolution of the figure. If one magnifies the figure around $k=0$, it can be seen that it is actually not a solution. 


\section{Conclusion}

Systems of $N$ contact potentials, particularly if these potentials are linear combinations of Dirac deltas, are usually solvable systems suitable for studying important features in nonrelativistic quantum mechanics, such as bound states, scattering transmission and reflection coefficients, threshold anomalies and resonances as quantum unstable states and virtual states (also called antibound states). In this study, we have shown that the Lippmann-Schwinger equation in the momentum space is a very appropriate tool for performing calculations in order to find the properties of the above-mentioned quantum features. In the case of $N$ Dirac deltas, we have found explicitly the solution of the Lippmann-Schwinger equation in terms of the matrix $\Phi$, which encodes all the information about the system. This method allows us to discuss the scattering problem (and bound states as well [31]) for arbitrary number of centres without solving the system of equations from the boundary conditions imposed at the location of the Dirac delta centres, and this substantially simplifies the calculations. Therefore, we were able to investigate the transmission, the reflection probabilities and the threshold anomalies for $N>2$ delta centres. Although we have explicitly found the scattering information of a very particular model, where a single particle interacts with finitely many singular potentials described formally by the Dirac delta functions, it can be applied to other systems as well by modelling them with a special arrangement of the Dirac delta potentials (see [5]). Hence, the methodology we have used here is of a general character.

\section{Acknowledgments}

The present work has been fully financed by TUBITAK from Turkey under the '2221Visiting Scientist Fellowship Programme'. We are very grateful to TUBITAK for this support. We also acknowledge Osman Teoman Turgut for the clarifying discussions and his interest in the present research. Finally, this work was also sponsored by the Spanish MINECO (MTM2014-57129-C2-1-P) and Junta de Castilla y León project no. VA057U16. We would also like to thank the anonymous reviewers whose comments improved this manuscript.

\section{Appendix A. Rigged Hilbert spaces}

This short appendix is devoted to a definition of the rigged Hilbert spaces (RHS), their most relevant properties and a brief account of their relevance in quantum systems with a continuous spectrum. A rigged Hilbert space is a tern of spaces with mutual relations of the form

$$
\Phi \subset \mathcal{H} \subset \Phi^{\times},
$$

where

(i) $\mathcal{H}$ is an infinite dimensional Hilbert space having a countable orthonormal basis.

(ii) $\Phi$ is a subspace of $\mathcal{H}$ with the following properties: (a) for each vector $f \in \mathcal{H}$ and each fixed positive number $\varepsilon>0$, no matter how small, there exists a vector $g \in \Phi$ such that its distance to $f$, measured in terms of the norm on $\mathcal{H}$, is smaller than $\varepsilon$, i.e. $\|g-f\|<\varepsilon$. We say that $\Phi$ is dense in $\mathcal{H}$. (b) The space $\Phi$ has its own topology, different from the norm topology inherited from $\mathcal{H}$ and having the important property that if we define the identity mapping i: $\Phi \longmapsto \mathcal{H}$ as $\mathrm{i}(g)=g$, for all $g \in \Phi$, then i must be continuous with the topologies on $\Phi$ and $\mathcal{H}$. This means that the topology on $\Phi$ has more open sets than 
those determined by the norm topology. Some extra properties are usually demanded for $\Phi$ in order to comply with the Dirac formulation of quantum mechanics, but they are too technical and we omit them, see [24, 25, 38, 39, 42-44].

(iii) Vectors $F \in \Phi^{\times}$are mappings from $\Phi$ into the field of complex numbers $\mathbb{C}$ satisfying two conditions. (a) Antilinearity: for each pair of vectors $f, g \in \Phi$ and each pair of complex numbers $\alpha, \beta \in \mathbb{C}$, we have that $F(\alpha f+\beta g)=\alpha^{*} F(f)+\beta^{*} F(g)$, where the star denotes complex conjugation. (b) Continuity: $F: \Phi \longmapsto \mathbb{C}$ has to be continuous with respect to the topologies on $\Phi$ and $\mathbb{C}$. Usually, these mappings are called functionals. The set of functionals is a vector space on the field of complex numbers that we call $\Phi^{\times}$the antidual space of $\Phi$. It is noteworthy that for any vector $f$ in the Hilbert space $\mathcal{H}$, there exists a unique functional $F_{f}$ defined as $F_{f}(g):=\langle g \mid f\rangle$ for any $g \in \Phi$. The identification of $F_{f}$ with $f$ for any $f \in \mathcal{H}$ justifies the inclusion $\mathcal{H} \subset \Phi^{\times}$. This latter fact suggests that the notation $F(g)$ for the action of $F$ on $g \in \Phi$ should be replaced by $\langle g \mid F\rangle$.

Concerning theoretical physics, the first application of the RHS was a rigorous treatment of the Dirac formulation of nonrelativistic quantum mechanics. In fact, the standard Hilbert space formulation does not include objects like plane waves that are used for the integral decompositions for observables with a continuous spectrum. The essential tool within this context is the Gelfand-Maurin theorem. We write this result below, where we have omitted some technicalities.

Some previous comments are in order. Assume that we have an RHS as in (A-1) and let $A$ be an operator on $\mathcal{H}$ with an adjoint $A^{\dagger}$. We say that $A$ reduces $\Phi$ or, equivalently, that $\Phi$ is invariant under the action of $A$, if for any vector $f \in \Phi$, then $A f \in \Phi$. Then, we write $A \Phi \subset \Phi$. Let us assume that the adjoint $A^{\dagger}$ reduces $\Phi$. Then, we may extend $A$ to the antidual $\Phi^{\times}$by making use of the duality formula:

$$
\left\langle A^{\dagger} g \mid F\right\rangle=\langle g \mid A F\rangle,
$$

valid for any $g \in \Phi$ and any $F \in \Phi^{\times}$. Although this extension of $A$ which acts on all $\Phi^{\times}$ should not be confused with the Hilbert space operator $A$, it is true that both are identical with respect to their action on vectors of $\Phi$. Then, in order not to burden the notation we use $A$ for both. If $A$ is Hermitian, (A-2) becomes

$$
\langle A g \mid F\rangle=\langle g \mid A F\rangle .
$$

Now, we may state the Gelfand-Maurin theorem, where we have omitted some technicalities $[42,45]$ :

Theorem (Gelfand-Maurin). Let A be a self-adjoint operator on an infinite dimensional Hilbert space. Then, a RHS exists as in (A-1) such that:

(i) The operator $A$ reduces $\Phi$, i.e. $A \Phi \subset \Phi$. In addition, $A$ is continuous under the topology on $\Phi$.

(ii) Assume that $A$ has a continuous spectrum, $\sigma(A)$. Then, for any $\lambda \in \sigma(A)$, there is a functional $F_{\lambda} \in \Phi^{\times}$, such that $A F_{\lambda}=\lambda F_{\lambda}$, where $A$ is the extension of $A$ into $\Phi^{\times}$given by the duality formula (A-3). It is customary to use $|\lambda\rangle$ instead of $F_{\lambda}$, so that $A|\lambda\rangle=\lambda|\lambda\rangle$.

(iii) The family of functionals $|\lambda\rangle$ for all $\lambda \in \sigma(A)$ is complete in the following sense: being given a function $h(\lambda)$, we may define for any pair $f, g \in \Phi$ :

$$
\langle f \mid h(A) g\rangle=\int_{\sigma(A)} h(\lambda)\langle f \mid \lambda\rangle\langle\lambda \mid g\rangle \mathrm{d} \lambda
$$


where $\langle f \mid \lambda\rangle=\langle\lambda \mid f\rangle^{*}$. For $h(\lambda) \equiv \lambda$, we have

$$
\langle f \mid A g\rangle=\int_{\sigma(A)} \lambda\langle f \mid \lambda\rangle\langle\lambda \mid g\rangle \mathrm{d} \lambda
$$

which is often written, omitting the arbitrary $f, g \in \Phi$ as

$$
A=\int_{\sigma(A)} \lambda|\lambda\rangle\langle\lambda| \mathrm{d} \lambda
$$

For $h(\lambda)=1$, we would have

$$
I=\int_{\sigma(A)}|\lambda\rangle\langle\lambda| \mathrm{d} \lambda
$$

where $I$ is the injection of $\Phi$ into $\Phi^{\times}$, i.e. $I(f) \in \Phi^{\times}$for any $f \in \Phi$.

Example. Let $\mathcal{H}$ be the Hilbert space of the square integrable functions on the whole real line, $L^{2}(\mathbb{R})$. Let $Q$ and $P$ be, respectively, the multiplication operator $(Q f)(x)$ and the derivation operator $(P f)(x)=-\mathrm{id} f(x) / \mathrm{d} x$. On $L^{2}(\mathbb{R}), Q$ and $P$ are called the position and the momentum operator, respectively. $Q$ and $P$ are both self adjoint on $L^{2}(\mathbb{R})$. We have a RHS implementing the Gelfand-Maurin theorem for both operators, when $\Phi$ is chosen to be the Schwartz space $S$.

The Schwartz space $S$ is a vector space of complex functions on the real line. Then, $f(x) \in S$ is a function $f: \mathbb{R} \longmapsto \mathbb{C}$, where $\mathbb{R}$ is the real line and $\mathbb{C}$ is the field of complex numbers, which in addition satisfies the following conditions:

(i) $f(x) \in S$ is differentiable at any point in any order.

(ii) All functions $f(x) \in S$ and their derivatives go to zero at infinity faster than the inverse of any polynomial.

Note that the definition implies that all the derivatives of any function in $S$ also belong to $S$. Skipping the topological details, one may prove that the triplet

$$
S \subset L^{2}(\mathbb{R}) \subset S^{\times}
$$

is a RHS [38, 39, 42]. The space $S^{\times}$is the space of tempered distributions seen as antilinear functionals. Both $Q$ and $P$ have a continuous spectrum covering the whole real line. For any $\lambda \in \mathbb{R}$, the functional $|\lambda\rangle$ for $Q$ is the Dirac delta $\delta(x-\lambda)$ and for $P$ the plane wave $\mathrm{e}^{-\mathrm{i} \lambda x}$.

\section{Appendix B. Bound states for $N$ Dirac delta potentials}

As we have mentioned in the introduction, the Dirac delta potentials can be represented by projections given by equation (4). Then, it is a simple exercise to show that the Schrödinger equation can be put into a matrix equation

$$
\sum_{j=1}^{N} \Phi_{i j}(E) \psi\left(a_{j}\right)=0,
$$

where

$$
\Phi_{i j}(E)=\left\{\begin{array}{cc}
1-\frac{m \lambda_{i}}{\hbar \sqrt{2 m|E|}} & \text { if } i=j, \\
-\frac{m \sqrt{\lambda_{i} \lambda_{j}}}{\hbar \sqrt{2 m|E|}} \exp \left(-\sqrt{2 m|E|}\left|a_{i}-a_{j}\right| / \hbar\right) & \text { if } i \neq j .
\end{array}\right.
$$


Here $E$ is real and negative. Actually, the matrix $\Phi$ given in equation (22) is exactly the analytical continuation of the above matrix $\Phi$. The bound state energies can be found from the nontrivial solution of the matrix equation for $\Phi$, that is, the bound state energies must satisfy $\operatorname{det} \Phi(E)=0$. This was explicitly shown in our recent paper [31]. Moreover, one can also imagine the following eigenvalue problem for the matrix $\Phi$, i.e.

$$
\Phi(E) A(E)=\omega(E) A(E) .
$$

Then, the bound state energies are the zeros of the eigenvalues $\omega$. This tells us that the eigenvalues of the linear Schrödinger equation $H \psi(x)=E \psi(x)$ are obtained through a nonlinear transcendental algebraic problem, $\omega(E)=0$.

\section{ORCID iDs}

Fatih Erman (10 https://orcid.org/0000-0003-0398-2225

\section{References}

[1] Kronig R de L and Penney W G 1931 Quantum mechanics of electrons in crystal lattices Proc. R. Soc. A 130499

[2] Pethick C J and Smith H 2008 Bose-Einstein Condensation in Dilute Gases (Cambridge: Cambridge University Press)

[3] Belloni M and Robinett R W 2014 The infinite well and Dirac delta function potentials as pedagogical, mathematical and physical models in quantum mechanics Phys. Rep. 54025

[4] Demkov Yu N and Ostrovskii V N 1988 Zero-range Potentials and Their Applications in Atomic Physics (New York: Plenum Press)

[5] Sahu B and Sahu B 2009 Phys. Lett. A 3734033

[6] Lapidus I R 1982 Resonance scattering from a double $\delta$-function potential Am. J. Phys. 50 663-4

[7] Senn P 1988 Threshold anomalies in one dimensional scattering Am. J. Phys. 56 916-21

[8] Berman P R 2013 Transmission resonances and Bloch states for a periodic array of delta function potentials Am. J. Phys. 81190

[9] Cordourier-Maruri G, De Coss R and Gupta V 2011 Transmission properties of the onedimensional array of delta potentials Int. J. Mod. Phys. B 25 1349-58

[10] Ahmed Z, Kumar S, Sharma M and Sharma V 2016 Revisiting double Dirac delta potential Eur. J. Phys. 37045406

[11] Patil S H 2009 Quadrupolar, triple $\delta$-function potential in one dimension Eur. J. Phys. 30629

[12] Barlette V E, Leite M M and Adhikari S K 2001 Integral equations of scattering in one dimension Am. J. Phys. 691010

[13] Lessie D and Spadaro J 1986 One dimensional multiple scattering in quantum mechanics Am. J. Phys. 54 909-13

[14] Hernández E, Jáuregui A and Mondragón A 2000 Degeneracy of resonances in a double barrier potential J. Phys. A: Math. Gen. 334507

[15] Antoniou I E, Gadella M, Hernández E, Jáuregui A, Melnikov Yu, Mondragón A and Pronko G P 2001 Gamow vectors for barrier wells Chaos Solitons Fractals 122719

[16] Espinosa M G and Kielanowski P 2008 Unstable quantum oscillator J. Phys: Conf. Ser. 128 012037

[17] Alvarez J J, Gadella M, Heras F J H and Nieto M 2009 A one-dimensional model of resonances with a delta barrier and a mass jump Phys. Lett. A 373 4022-7

[18] Alvarez J J, Gadella M and Nieto L M 2011 A study of resonances in a one dimensional model with singular Hamiltonian and mass jump Int. J. Theor. Phys. 50 2161-9

[19] Alvarez J J, Gadella M, Lara L P and Maldonado-Villamizar F H 2013 Unstable quantum oscillator with point interactions: Maverick resonances, antibound states and other surprises Phys. Lett. A 3772510

[20] Taylor J R 1972 Scattering Theory: The Quantum Theory of Non-Relativistic Collisions (New York: John Wiley and Sons) 
[21] Bohm A 1978 The Rigged Hilbert Space and Quantum Mechanics, Springer Lecture Notes in Physics 78 (New York: Springer)

[22] Appel W 2007 Mathematics for Physics and Physicists (Princeton, NJ: Princeton University Press)

[23] Gieres F 2000 Mathematical surprises and Dirac formalism in quantum mechanics Rep. Prog. Phys. 631893

[24] Antoine J P 1969 Formalism and symmetry problems in quantum mechanics. i. General Dirac formalism J. Math. Phys. 1053

[25] Gadella M and Gómez F 2002 A unified mathematical formalism for the Dirac formulation of quantum mechanics Found. Phys. 32815

[26] Gadella M and Gómez G 2002 The Lippmann-Schwinger equations in the rigged Hilbert space J. Phys A: Math. Gen. 358505

[27] de la Madrid R 2006 The rigged Hilbert space approach to the Lippmann-Schwinger equation: II. The analytic continuation of the Lippmann-Schwinger bras and kets J. Phys. A: Math. Gen. 393981

[28] Ash R B and Novinger W P 2007 Complex Analysis (New York: Dover)

[29] Griffiths D J 2005 Introduction to Quantum mechanics (Hoboken, NJ: Pearson Education)

[30] Rorres C 1974 Transmission coefficients and eigenvalues of a finite one-dimensional crystal SIAM J. Appl. Math. 27303

[31] Erman F, Gadella M, Tunalı S and Uncu H 2017 A singular one-dimensional bound state problem and its degeneracies Eur. Phys. J. Plus 132352

[32] Nussenzveig H M 1972 Causality and Dispersion Relations (New York: Academic)

[33] Bohm A 1986 Quantum Mechanics: Foundations and Applications (Berlin: Springer)

[34] Galindo A and Pascual P 1990 Quantum mechanics, (New York: Springer)

[35] Blatt J M and Weisskopf V F 1952 Theoretical Nuclear Physics (New York: Wiley)

[36] Michel N, Nazarevicz W, Ploszajckzak M and Vertse T 2009 Shell model in the complex energy plane J. Phys. G: Nucl. Part. Phys. 36013101

[37] Id Betan R, Liotta R J, Sandulescu N and Vertse T 2004 A shell model representation with antibound states Phys. Lett. B $\mathbf{5 8 4} 48$

[38] Bohm A and Gadella M 1989 Dirac Kets, Gamow Vectors and Gelfand Triplets, Springer Lecture Notes in Physics vol 348 (New York: Springer)

[39] Civitarese O and Gadella M 2004 Physical and mathematical aspects of Gamow states Phys. Rep. 39641

[40] Nussenzveig H M 1959 The poles of the $S$-matrix of a rectangular potential well of barrier Nucl. Phys. 11499

[41] Zavin R and Moiseyev N 2004 One-dimensional symmetric rectangular well: from bound to resonance via self-orthogonal virtual state J. Phys. A: Math. Gen. 374619

[42] Gelfand I M and Vilenkin N Y 1964 Generalized Functions: Applications to Harmonic Analysis (New York: Academic)

[43] Roberts J E 1966 Rigged Hilbert spaces in quantum mechanics Commun. Math. Phys. 3 98-119

[44] Melsheimer O 1974 Rigged Hilbert space formalism as extended mathematical formalism for quantum systems I. General theory J. Math. Phys. 15 902-16

[45] Maurin K 1968 General Eigenfunction Expansions and Unitary Representations of Topological Groups, Monografie Matematyczn 48 (Warszawa: PWN-Polish Scientific Publishers) 